\title{
a pertura
}

Volumen 10, número 1, pp. 149-163. | Universidad de Guadalajara

\section{Redefinición del} "aprendizaje en red" en la cuarta revolución industrial

Alexandro Escudero Nahón*

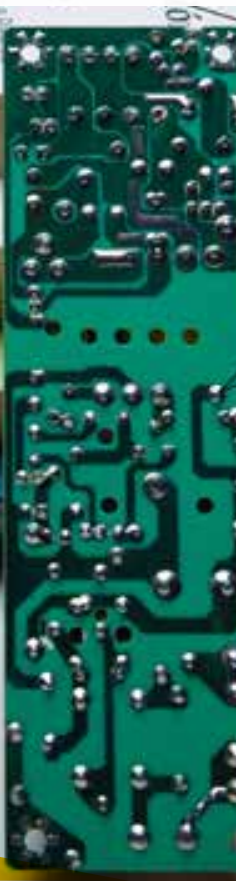

\section{RESUMEN}

La "cuarta revolución industrial" provocará una transformación en los escenarios educativos porque incorporará redes ciberfísicas en la producción, logística y consumo de bienes y servicios. La teoría educativa ha abordado tradicionalmente los escenarios educativos tecnologizados con categorías de análisis propias de la tercera revolución industrial y es necesario superar esos lastres conceptuales. Este texto propone el concepto de "aprendizaje en red" y toma como punto de partida la ontología poshumanista y la epistemología de la teoría del actor-red. Así, el aprendizaje sería un proceso que asocia agencias humanas y no humanas por igual, que opera sin centro ni periferia, y cuyo objetivo es garantizar su autorregulación y persistencia porque en cuanto las agencias dejan de operar, la red de aprendizaje desaparece. El aprendizaje es, en sí mismo, una red heterogénea en funcionamiento. Asimismo, en este texto se identifican algunas agencias humanas y no humanas básicas que permiten constituir redes de aprendizaje, fortalecerlas o destruirlas. Además, se justifica por qué la investigación educativa debería crear datos de manera inductiva y construir categorías de análisis en forma abductiva para identificar nuevos objetos de estudio en los escenarios de aprendizaje de la cuarta revolución industrial, que se caracterizan por ser híbridos, ambiguos y contingentes.

\section{Palabras clave}

Teoría educativa, poshumanismo, teoría del actor-red, cuarta revolución industrial, aprendizaje en red, filosofía de la educación

\footnotetext{
* Doctor en Educación. Profesor-investigador de tiempo completo. Universidad Autónoma de Querétaro. México.
} 


\section{Redefinition of "Network learning" \\ in the fourth industrial revolution}

\section{Abstract}

The "fourth industrial revolution" will provoke a transformation in educational scenarios because it will incorporate cyber-physical networks in the production, logistics and consumption of goods and services. Educational theory has traditionally approached technological educational scenarios with categories of analysis typical of the third industrial revolution and it is necessary to overcome these conceptual weights. This paper proposes the concept of "network learning" taking as a starting point the posthumanist ontology and the epistemology of the actor-network theory. Thus, learning would be a process that associates human and non-human agencies alike, which operates without center nor periphery, and whose objective is to guarantee self-regulation and persistence because as soon as the agencies stop operating, the learning network disappears. Learning is, in itself, a heterogeneous network in operation. Likewise, this paper identifies some basic human and non-human agencies that allow learning networks to be established, strengthened or destroyed. In addition, it is justified why educational research should create data through an inductive manner and construct categories of analysis abductively to identify new objects of study in the learning scenarios of the fourth industrial revolution, which are characterized by being hybrid, ambiguous and contingent.

Keywords

Educational theory, posthumanism, actor-network theory, fourth industrial revolution, network learning, philosophy of education

\section{INTRODUCCIÓN}

$\mathrm{D}^{2}$ e acuerdo con la literatura especializada, estamos en los albores de la cuarta revolución industrial y, tras lo sucedido en las tres revoluciones industriales anteriores, se esperan cambios radicales en nuestra manera de vivir, trabajar, estudiar, consumir y relacionarnos unos con otros (Schwab, 2015, 2016; World Economic Forum, 2016).

La primera revolución industrial surgió a finales del siglo XVIII en el Reino Unido, con la irrupción de la energía de vapor, la mecanización de la agricultura, la manufactura y el transporte (Daemmrich,
2017). La segunda revolución industrial se consolidó en Estados Unidos de América a principios del siglo XX. En este caso, la tecnología disruptiva fue la energía eléctrica, que permitió la producción en serie (Hintz, 2011; MacLeod, 2016). La tercera revolución industrial fue detonada desde mediados del siglo pasado con la invención de los semiconductores, las plataformas digitales y el auge de las computadoras personales (Hermann, Pentek \& Otto, 2016).

A diferencia de las tres revoluciones anteriores, la cuarta no se define por la emergencia de una tecnología disruptiva específica, sino por la convergencia de varias tecnologías digitales, físicas y biológicas, 
como la inteligencia artificial, la inteligencia aumentada, la robótica, la impresión $3 \mathrm{D}$, el cloud computing, el big data, el "internet de las cosas" o la nanotecnología (Rose, 2016). Esta confluencia genera redes ciberfísicas. Lejos de ser un escenario de ciencia ficción, estas redes ya funcionan en escenarios laborales, financieros o de ocio, con capacidades de autorregulación, toma de decisiones propias y con mínima o nula intermediación de seres humanos (Roland Berger, 2016).

La asociación entre varias redes ciberfísicas está generando proyectos estimulantes como "ciudades inteligentes", "fábricas 4.0", "economías verdes", etcétera (Tecnalia, 2017), pero también está provocando desafíos para que las personas adquieran las competencias profesionales necesarias para competir en el mercado laboral actual (Devezas, Leitao \& Sarygulov, 2017; Lorenz et al., 2016; Lorenz et al., 2015).

La tercera revolución industrial tuvo un enfoque orientado al producto y por eso se requerían competencias profesionales centradas en automatizar procesos y máquinas individuales para introducir mejoras en el producto (Hermann et al., 2016; Kagermann, Wahlster \& Helbig, 2013). Ahora, la cuarta revolución industrial está creando un enfoque centrado en ecosistemas digitales, es decir, está generando modelos de negocios innovadores basados en la interconexión de millones de consumidores, máquinas, productos y servicios. Por eso, se requieren nuevas competencias profesionales que permitan aplicar mejoras a lo largo de toda la cadena de valor (Slama et al., 2015); por ejemplo, el pensamiento crítico, la creatividad, la inteligencia emocional, etcétera (Morita-Alexander, García-Ramírez \& Escudero-Nahón, 2016). Al mismo tiempo, se requieren nuevas competencias pro- fesionales técnicas, esto es, conocimientos propios de las redes ciberfísicas y los ecosistemas digitales (Adams et al., 2017; OCDE, 2017; UNESCO, 2015; World Economic Forum, 2016).

De acuerdo con el estudio "50 innovaciones educativas en América Latina. Graduate XXI, un mapa del futuro" (Rivas \& Delgado, 2016), varias innovaciones educativas altamente tecnologizadas están consolidándose en nuestro continente. Destacan, por ejemplo, el aprendizaje adaptativo, que usa la inteligencia artificial y el big data; los servicios educativos integrales en red, que utilizan el cloud computing y el internet de las cosas; la gamificación de la educación, que basa su originalidad en la noción de "persistencia en línea", como lo hacen los videojuegos de internet o las plataformas de realidad virtual; el aprendizaje híbrido y móvil, que pone a disposición del aprendiz varias tecnologías en línea y herramientas tradicionales.

La investigación educativa ha abordado estas innovaciones educativas con diversas categorías de análisis; por ejemplo, e-learning, entorno personal de aprendizaje,

\section{La cuarta revolución industrial}

\section{está creando un enfoque}

\section{centrado en ecosistemas}

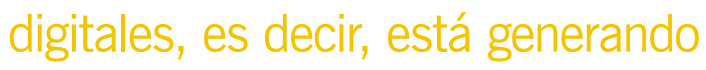

modelos de negocios innovadores

basados en la interconexión

de millones de consumidores,

máquinas, productos y servicios 
entorno virtual de enseñanza-aprendizaje, educación mediada por tecnología, aprendizaje en red, entre otras (Gros, 2012; Hsu et al., 2012; Hung, 2012). El concepto "aprendizaje en red" es en particular interesante porque hace referencia a las conexiones técnicas y humanas que los estudiantes realizan para obtener buenos resultados académicos en ambientes de estudio altamente tecnologizados. Esta expresión no es original, porque, en realidad, independientemente de cualquier época histórica, las personas siempre han creado un entramado de conexiones para aprender, pero solo fue posible reflexionar al respecto cuando la tecnología digital permitió ciertas conexiones inusuales que superan las limitaciones temporales y físicas para acceder a nuevas fuentes de información y a nuevos referentes de aprendizaje (Jiménez, Bustamante \& Albornoz, 2015; Pedersen, 2010).

Sin embargo, el aprendizaje en red presenta ciertas limitaciones conceptuales para abordar estas innovaciones educativas, ya que es heredero de los principios ontológicos y epistemológicos del humanismo moderno, que se caracteriza por ser antropocéntrico y dicotómico (Pedersen, 2010). Por eso, asume que la tecnología y la educación son esferas bien diferenciadas y autónomas entre sí (Jiménez et al., 2015; Thomas \& Buch, 2013), y coloca con premura al aprendiz en el centro del proceso educativo y lo encumbra sobre todos los demás elementos no humanos que participan en el aprendizaje (Carlson, 2015).

Lo anterior provoca que este término sea poco útil para estudiar la innovación educativa en escenarios altamente tecnologizados, donde existe un pronunciado protagonismo de elementos no humanos que operan sin centro ni periferia, es decir, como una red (Knox, 2016; Minerva, Biru \& Rotondi, 2015; Rivas \& Delgado, 2016).

Por primera vez en la historia se ha podido identificar la emergencia de una revolución industrial. Por eso, la investigación educativa tiene el desafío y la oportunidad de superar la visión humanista moderna que actualmente entraña el aprendizaje en red y redefinir esta categoría de análisis con el objeto de explicar la relación de la tecnología educativa con los procesos de aprendizaje (Baygin et al., 2016; Escudero, 2016a).

Este texto argumenta que es necesario realizar una propuesta ontológica poshumanista y una propuesta epistemológica basada en la teoría del actor-red para redefinir el concepto de aprendizaje en red. Posteriormente, propone dos métodos de investigación para el estudio de este tipo de aprendizaje: la obtención de datos de manera inductiva y la construcción de categorías de análisis en forma abductiva. El objetivo es esbozar un procedimiento de investigación para rastrear qué elementos humanos y no humanos se asocian para crear agencias y cómo se originan las redes de aprendizaje, cómo se fortalecen, cómo se asocian a otras redes y cómo desaparecen.

\section{ANTROPOCENTRISMO Y DICOTOMIÍA EN EL HUIMANISIMO MODERNO}

Los primeros registros del vocablo humanismo datan de la humanitas latina. En ese período, el término hacía referencia a una distinción entre la cultura y la educación que debía recibir el hombre libre y que no era propia, de ningún modo, del "hombre bárbaro" (Arbea, 2002). A lo largo de la historia, se transformó en diferentes tipos de humanismo, como el clásico, el medieval, el ilustrado, el existencialista, etcétera (Echeverría, 2006; Kakkori \& Huttunen, 2010), pero todos ellos dotaron a "lo humano" de cuatro características (Espinosa, 2016; Knox, 2016): esencialismo, universalismo, autonomía y trascendencia. En efecto, el humanismo es una distinción, explícita o velada, que el humano hace de sí mismo basada en una supuesta condición esencial que lo separa y lo hace distinto, mejor y más importante que "lo no humano".

El humanismo propiamente moderno surgió en el siglo XVII en Europa. A diferencia de los humanismos anteriores, este estuvo insuflado por el pensamiento ilustrado y por un peculiar 

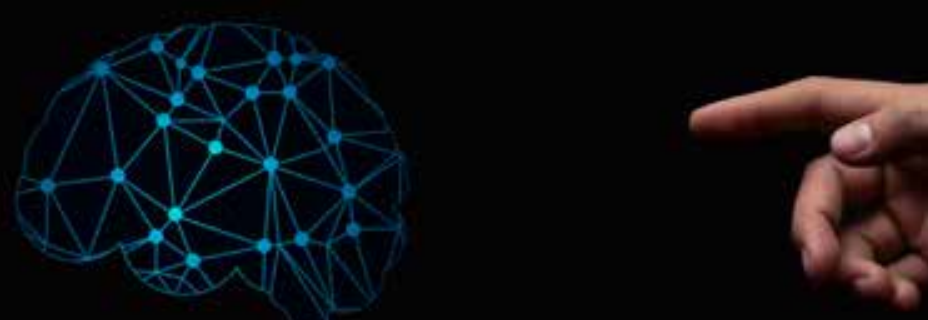

optimismo sobre la facultad racional del ser humano (Chernilo, 2017). Por eso, el proyecto moderno se basó en la promesa de que el dominio de la ciencia y la tecnología liberaría a la humanidad de sus atavismos premodernos (Dolphijn \& Van der Tuin, 2011). El humanismo moderno, sin embargo, fundó su ontología sobre una tramposa sinécdoque (Weaver, 2010). Al postular que "lo humano es la medida de todas las cosas”, es decir, al hacer pasar la parte por el todo, produjo un mundo antropocéntrico que, por definición: a) está jerarquizado, pues sobreestima la agencia de "lo humano" y desprecia la agencia de "lo no humano"; b) separa la ontología de la epistemología, pues separa al que aprende de lo que aprende, y de con qué aprende; c) divide y clasifica a las disciplinas del conocimiento de acuerdo con un objeto de estudio antropocéntrico, esto es, supone que "lo humano" es un sujeto de estudio con atributos bien definidos y sustancialmente distinto a "lo no humano".

Para sostener el antropocentrismo, el humanismo moderno debió recurrir a otra argucia: la dicotomía. Primero, argumentó que, debido a su esencia racional, "lo humano" mantenía una re- lación disruptiva con "lo no humano"; segundo, simplificó la complejidad de las interconexiones que "lo humano" mantiene con el mundo en dos categorías (humano/no humano); y tercero, este ordenamiento simbólico dicotómico implicó siempre una injusta desvaloración de lo "no humano" (St. Pierre, 2013).

La teoría educativa moderna es heredera del humanismo moderno y, por eso, su punto de partida siempre es un paradigma provincial limitante que coloca en el centro a "lo humano" como único sujeto cognoscente, con atributos esenciales bien definidos, separado de lo que aprende y de las cosas que usa para aprender (Snaza \& Weaver, 2015). Esa herencia también imprime otra limitación: la teoría educativa parte de supuestos dicotómicos y, por muy complejas que sean las interconexiones entre los elementos en los escenarios educativos, se les dota de límites bien definidos, de atributos esenciales y de una injusta desvaloración de, por ejemplo, "el objeto" ante "el sujeto", "el cuerpo" ante "la mente", "el entorno" ante "la persona", "la tecnología" ante "la educación", en fin, desvalora "lo no humano" ante "lo 
humano" (González, 2015). Justamente porque el humanismo moderno todavía domina el discurso de la tecnología educativa, es que este ámbito de estudio se aborda en forma incorrecta como dos mundos diferentes en términos ontológicos, como esferas dicotómicas y autónomas entre sí (Thomas \& Buch, 2013).

Desde la segunda mitad del siglo XX, los efectos indeseables del humanismo moderno están amenazando, de manera irónica, la supervivencia de nuestra especie. La polución, la desigualdad y la discriminación han provocado cierto desencanto sobre la promesa del proyecto moderno y han catalizado un ímpetu por hacer reformulaciones ontológicas y epistemológicas que conduzcan a nuevas conceptualizaciones de lo humano. El protagonista principal de esta convulsión teórica es el poshumanismo.

El poshumanismo es un pensamiento compuesto por varias tendencias teórico-conceptuales, como el poshumanismo crítico, el poshumanismo antiantropocéntrico, el transhumanismo y el poshumanismo analítico, cuyo objetivo común es reformular una nueva ontología que dé lugar a una definición distinta de humano (Braidotti, 2013; Skageby et al., 2016; Weaver, 2010; Wolfe, 2010). Por eso, la aplicación del poshumanismo a la educación aboga por una ruptura

\section{Desde la segunda mitad del} siglo XX, los efectos indeseables del humanismo moderno están amenazando, de manera irónica, la supervivencia de nuestra especie. La polución, la desigualdad y la discriminación han provocado cierto desencanto de la visión antropocéntrica y dicotómica e introduce un pensamiento complejo que a) des-centra lo humano y lo coloca en el mismo nivel de importancia que lo no humano al momento de aprender (Wolfe, 2010); b) admite que lo humano y lo no humano constituyen al mundo, se constituyen en interdependencia y, por lo tanto, sus agencias son igualmente importantes para aprender (Braidotti, 2013); c) deja de suponer que el que aprende invariablemente es un humano (Snaza \& Weaver, 2015); y d) admite que lo no humano siempre ha sido condición de posibilidad del aprendizaje y no solo un accesorio de ese proceso.

De esta manera se supera el sentido artefactual y simplificado de la tecnología educativa, referido solo a las herramientas que sirven para enseñar y aprender, y se construye una versión más compleja que incluye a todos los elementos humanos y no humanos en procesos dinámicos (DeLanda, 2006; Usher \& Edwards, 2005). Lo anterior obliga a construir un nuevo relato epistemológico para el estudio de los escenarios educativos tecnologizados, que se caracterizan por estar pletóricos de elementos heterogéneos que mantienen distintas conexiones entre sí, como el software, las redes sociales, el hardware, la señal de internet, la inteligencia artificial, la inteligencia aumentada, el internet de las cosas, las plataformas de gestión del aprendizaje y, por supuesto, también los humanos.

A partir de la segunda mitad del siglo XX, la debacle de la visión moderna y positivista en las ciencias sociales provocó varias reformulaciones teóricas y conceptuales. Una de las más relevantes fue la bifurcación que experimentó la sociología tradicional con la emergencia de la "sociología de las asociaciones” (Devenin \& Henríquez, 2011). Esta propuesta argumentó que la sociología tradicional había sufrido un menoscabo en la capacidad para abordar, describir y explicar sus objetos de estudio porque daba por sentado que la sociedad existe, en sí misma, de manera esencial (Latour, 2008).

De acuerdo con esa crítica, la sociología tradicional habría provocado, por lo menos, dos efectos indeseables (Pignuoli-Ocampo, 2014): 
a) tomó el término "sociedad" como explicación de lo que debía ser explicado y confundió, así, la causa con el efecto: el resultado fue un déficit en su capacidad explicativa; y b) como dio por sentado que la sociedad existe esencialmente, entonces procuró describir las propiedades de esa "sustancia” social y perdió capacidad de reacción ante el dinamismo del objeto de estudio.

En contraposición, la "sociología de las asociaciones" propuso un andamiaje teórico conceptual que revitalizó los estudios sociales: la teoría del actor-red (TAR) (Latour, 2007, 2008, 2009). La TAR se consolidó en la década de los ochenta del siglo XX y desde entonces es un referente para los estudios que abordan con seriedad el papel de la ciencia y la tecnología en relación con la noción de sociedad (Jackson, 2015).

Uno de los principios epistemológicos más audaces de la TAR es que la sociedad, en sí misma, no existe, por lo menos no como una sustancia esencial en la que suceden cosas sociales (léase la ironía). Tampoco es un contexto homogéneo; mucho menos una prerrogativa de los humanos sobre lo no humano. Para la TAR, lo social es todo aquello que está asociado como una red (Harman, 2009). Así, las asociaciones hacen a la sociedad, y no al revés. Este giro copernicano en el objeto de estudio permite definir que la sociedad es un movimiento, un proceso de ensamblado, un fluido circulante que pone en contacto a múltiples elementos y agencias. Estos elementos y agencias, en general, son humanos y no humanos.

Por eso, el principio epistemológico de la TAR es útil para redefinir el término aprendizaje en red. Sin embargo, antes vale la pena aclarar que, a diferencia de otras definiciones de red, la TAR postula un carácter performativo de red, y no uno ostentativo; un carácter híbrido de los elementos que la constituyen; una concepción amplia del término agencia; y una simetría entre los elementos humanos y los no humanos (Latour, 2008). De este modo, para la TAR:

- La red es performativa y no ostentativa. Una red es tal en la medida que los elementos
Para que una red sea potente, necesariamente debe garantizar

\section{la persistencia de sus agencias.}

\section{Los elementos no humanos son}

fundamentales para cumplir con

este fin. Por eso, la importancia

de los elementos humanos y no

humanos es simétrica en la red

que la componen ejercen sus agencias; en cuanto esas agencias dejan de operar, la red desaparece. Por eso, ningún elemento de la red ostenta en sí mismo, esencialmente, ninguna agencia; es necesario que sucedan asociaciones para que las agencias generen una red.

- La red es híbrida. Si los elementos humanos y no humanos logran asociarse, fundan unidades híbridas susceptibles de ser analizadas como elementos "casi humanos" o elementos "casi no humanos".

- Existen múltiples tipos de agencias en una red y no todas las realiza el ser humano. Una agencia no es solo la capacidad que tienen los elementos de la red de generar transformaciones, sino también su capacidad de recibir esas influencias, como si se tratasen de un blanco móvil.

- Para que una red sea potente, necesariamente debe garantizar la persistencia de sus agencias. Los elementos no humanos son fundamentales para cumplir con este fin. Por eso, la importancia de los elementos humanos y no humanos es simétrica en la red, pues su valor no radica en su condición humana, sino en la capacidad que tengan para garantizar que nada amenace la correcta operación de las agencias. 
El ingreso a la red tiene por objetivo rastrear con meticulosidad las agencias que surgen cuando elementos humanos y no humanos están en actividad. Este proceso de ingreso a la red es inductivo porque suspende las categorías analíticas previamente aprendidas

Así, el aprendizaje en red puede definirse de la siguiente manera: el aprendizaje es, en sí mismo, una red en funcionamiento. Esta red requiere asociar varios elementos y agencias humanas y no humanas correctamente. Esta red no tiene un carácter esencialista, sino que existirá en la medida que sus elementos humanos y no humanos ejerzan sus agencias apropiadamente. El aprendizaje en red es performativo y en cuanto las múltiples agencias dejan de operar correctamente, la red desaparece. Cuando el aprendizaje en red es potente y tiene resultados innovadores, es capaz de ensamblarse con otras redes, puede sostenerse por mucho tiempo, puede fortalecerse y da la impresión que atrae, influye y transforma casi todo.

Si la investigación educativa admitiera que las asociaciones fundan lo social, y no al revés, entonces estaría en condición de abordar el proceso de aprendizaje como una red. Sería capaz de rastrear las agencias que los elementos humanos y no humanos provocan al estar asociados en una red de aprendizaje. Mejoraría su capacidad explicativa y su velocidad de reacción ante los escenarios educativos actuales (Echeverría \& González, 2009). Se abriría un horizonte de conceptualizaciones que sí podrían dar cuenta de los actores emergentes que están constituyendo a la tecnología educativa. Por lo anterior, el objetivo de la investigación educativa, desde esta perspectiva, sería rastrear qué elementos humanos y no humanos se asocian para generar agencias, cuáles son las agencias más importantes, cuáles son los principales problemas que tienen las redes de aprendizaje para surgir, estabilizarse, persistir, fortalecerse, o por qué desaparecen (Escudero, 2016b).

\section{OBJETIVO DE LA INVESTIGACIÓN EDUCATIVA DESDE LA PERSPECTIVA DE LA TAR \\ Rastrear múltiples agencias de manera inductiva}

La investigación positivista, que predominó en los estudios de educación hasta la segunda mitad del siglo XX, tenía como objetivo principal verificar hipótesis. Por eso, la mayoría de los estudios fueron realizados con el método hipotético-deductivo, que se caracteriza por delimitar con precisión un problema de estudio, construir un marco teórico denso con categorías de análisis predefinidas, y diseñar instrumentos de recolección de datos antes de ingresar al campo de estudio.

El método de investigación que aquí proponemos es diametralmente opuesto. El ingreso a la red tiene por objetivo rastrear con meticulosidad las agencias que surgen cuando elementos humanos y no humanos están en actividad. Este proceso de ingreso 
a la red es inductivo porque suspende las categorías analíticas previamente aprendidas; no parte de un diseño de investigación previo al ingreso a la red ni se acompaña de ningún instrumento de obtención de datos prediseñado, sino que todo eso se consolida a lo largo del estudio (Bryant, 2017; Gibson \& Hartman, 2014; Holton \& Walsh, 2016).

Si se acepta la premisa de que no existen objetos de estudio previamente definidos, con atributos inherentes o límites definidos en un proceso de aprendizaje en red, sino que los elementos y sus agencias se ensamblan en situación, y solo el funcionamiento apropiado de este ensamblaje instala la red, entonces se puede admitir que los objetos de estudio en la red son híbridos, contingentes, contradictorios, ambiguos, paradójicos. No obstante, sí es posible rastrear la actividad de la red o, por lo menos, los sedimentos de esa actividad (Fenwick \& Edwards, 2010, 2011; Fenwick, Edwards \& Sawchuk, 2011; Law, 2004).

El acento del método inductivo no está puesto en verificar la existencia o ausencia de categorías analíticas previamente diseñadas, sino en ser sensible a los datos que genera la actividad de los elementos y sus agencias en la red. Esta sensibilidad podría ser estimulada al intentar responder dos sencillas preguntas: ¿cuál es el principal problema para que se realicen las agencias en la red de aprendizaje? y ¿́cómo intentan los elementos de la red resolver ese problema?

Las técnicas e instrumentos tradicionales de obtención de información siguen siendo útiles, pero ahora el objetivo es identificar las ambigüedades, contingencias, paradojas, controversias, etcétera, con las cuales suelen actuar los elementos y las agencias de una red. Las entrevistas en profundidad, las conversaciones informales, los grupos de enfoque, etcétera, son técnicas muy adecuadas para obtener información de las agencias humanas de una red; la observación, la inmersión, los registros multimedia, etcétera, suelen ser útiles para registrar las agencias de los actores no humanos (Fenwick \& Edwards, 2010). La información lograda con estos instrumentos, sin embargo, no es suficiente para crear categorías de análisis. Es necesario aplicar otro método para superar la sola descripción del problema y proponer una explicación conceptual.

\section{Construir categorías analíticas de manera abductiva}

La abducción es un proceso cognitivo que puede estimular la creación de categorías analíticas porque integra, evalúa y, en ocasiones, desintegra de manera constante varias y diversas hipótesis a lo largo de la investigación a fin de dar sentido a hechos que son aparentemente desconcertantes. Locke et al. (2008) lo esquematizaron de la siguiente manera: la deducción es capaz de decir que "algo debe ser así"; la inducción demuestra que "algo es así", pero la abducción sugiere que "algo podría ser así". A diferencia del método deductivo de investigación, que diseña una hipótesis antes de ir al campo de estudio, la abducción integra y desintegra en forma constante varias hipótesis usando categorías de análisis construidas en la red para explicar el problema en cuestión (Dunne \& Dougherty, 2015). El resultado de la abducción es la explicación de un problema a partir de la reiterada creación de hipótesis que se comprueban en la red o que se desintegran para crear nuevas hipótesis (Pape, 1999; Reichertz, 2007).

Una de las implicaciones metodológicas de la redefinición del concepto aprendizaje en red con base en la TAR es que la red de 
estudio no puede ser concebida desde un punto de vista esencial, que existe por sí misma. No: la red de aprendizaje existirá siempre que elementos humanos y no humanos se asocien y generen agencias. Cuando esto deja de suceder, la red de aprendizaje desaparece. Por lo tanto, el ingreso a la red de estudio requiere identificar, antes que nada, si están operando algunas agencias y cuál es la que funciona con más intensidad.

A continuación, presentamos una propuesta de agencias generales que conformarían el aprendizaje en red y que han sido adaptadas del procedimiento general de las redes ciberfísicas propias de la cuarta revolución industrial (Hermann et al., 2016) (ver figura).

En el nivel descriptivo, es necesario identificar qué elementos humanos y no humanos se asocian para generar cada una de estas agencias. Como explicamos en la figura, algunas de estas agencias las realizan mejor los elementos no humanos, otras los humanos, y otras se efectúan mejor en colaboración de ambos. Las agencias operan en la red sin orden preestablecido, de manera continua, y serán efectivas siempre y cuando sean performa- tivas, es decir, siempre que mantengan bien asociados a varios elementos de la red.

Las agencias más rutinarias, como captar datos con sensores e intercambiarlos, han sido ya sustituidas por robots que, con base en ciertos materiales sensoriales y algoritmos, desempeñan una labor mucho más eficiente que los humanos. Sin embargo, existen otras agencias en las que la colaboración entre humanos y no humanos ha resultado de gran utilidad, por ejemplo, la producción de información pertinente, su interpretación a partir de algunos criterios adecuados a la situación y el registro de este tipo de conocimiento. Por último, las agencias de intuición, reflexión e innovación son, hasta ahora, predominantemente humanas.

En el nivel conceptual, es necesario explicar con una categoría de análisis cuáles problemas impiden que los elementos humanos y no humanos se asocien para generar y sostener cada una de estas agencias al tiempo que se registra cómo logran los elementos de la red superar ese problema. Por eso, es conveniente una descripción general de las agencias que constituyen las redes ciberfísicas en la cuarta revolución industrial (ver tabla).

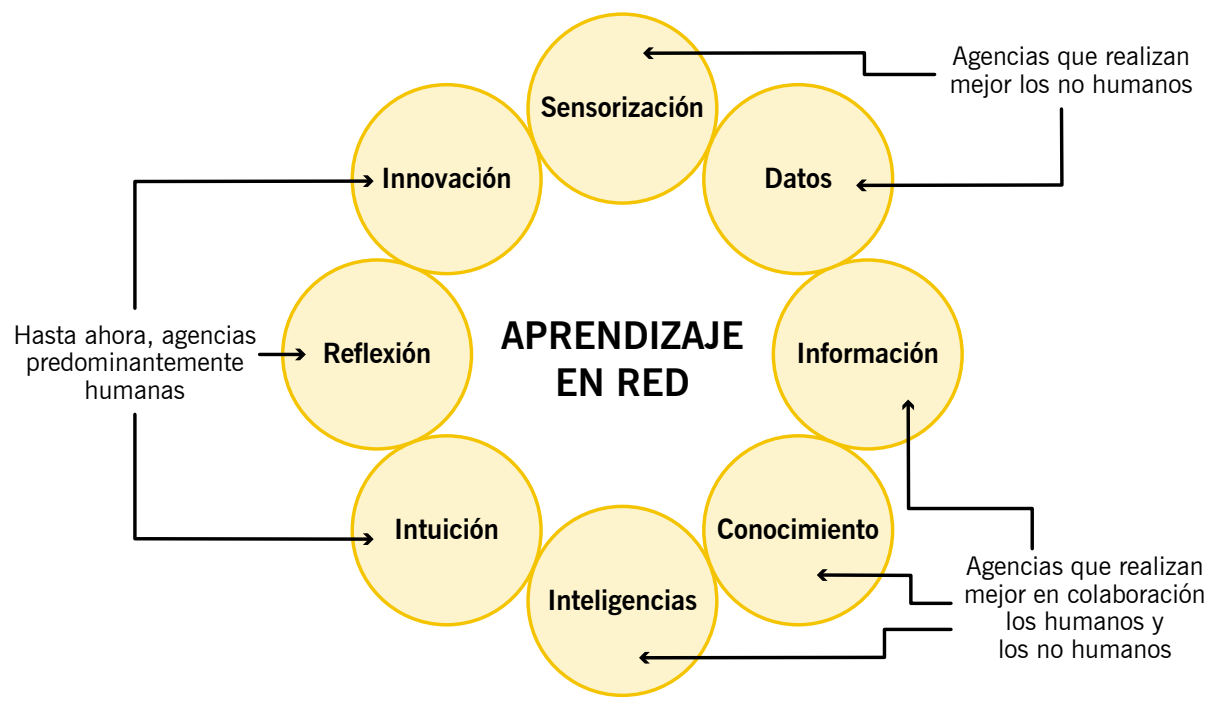

Figura. Agencias generales que se realizan en el aprendizaje en red.

Fuente: elaboración propia. 
Tabla. Descripción de las fases del aprendizaje en red

\begin{tabular}{|l|l|}
\hline \multicolumn{1}{|c|}{ ACTIVIDAD } & \multicolumn{1}{c|}{ DESCRIPCIóN } \\
\hline Sensorización & $\begin{array}{l}\text { Captar con la mayor precisión posible los estímulos y las variaciones del ambiente, como el } \\
\text { movimiento, la velocidad, la temperatura, la humedad, los sonidos, la imagen, etcétera. Es- } \\
\text { tos estímulos deben ser traducidos en datos para que puedan servir para tomar decisiones }\end{array}$ \\
\hline Intercambio de datos & $\begin{array}{l}\text { El intercambio oportuno de datos y su interpretación dentro de un contexto y con ciertos } \\
\text { criterios los convierten en información valiosa }\end{array}$ \\
\hline $\begin{array}{l}\text { Producción de } \\
\text { información }\end{array}$ & $\begin{array}{l}\text { La información tratada con destrezas adecuadas se convierte en categorías de análisis. } \\
\text { La aplicación rigurosa de estas categorías en situaciones problemáticas específicas se } \\
\text { convierte en conocimiento }\end{array}$ \\
\hline Conocimiento & El conocimiento con un propósito propulsa las inteligencias \\
\hline Inteligencias & $\begin{array}{l}\text { La aplicación reiterativa y creativa de las inteligencias para explicar o resolver un problema } \\
\text { específico provoca varias acciones, pero se optimizan los recursos invertidos en esta apli- } \\
\text { cación con la intuición }\end{array}$ \\
\hline Intuición & $\begin{array}{l}\text { La intuición agrupa de manera dinámica la experiencia, las inteligencias, la imaginación, la } \\
\text { creatividad, etcétera, pero se regula gracias a la reflexión }\end{array}$ \\
\hline Innovación & $\begin{array}{l}\text { La reflexión es capaz de evaluar desde distintos puntos de vista el proceso a través del } \\
\text { cual se realizaron todas las agencias anteriores. Asimismo, evalúa la pertinencia, utilidad y } \\
\text { originalidad de los resultados para reconocer el grado de innovación }\end{array}$ \\
\hline
\end{tabular}

Fuente: elaboración propia.

Esta propuesta de agencias generales del aprendizaje en red resultaría útil para identificar en los escenarios educativos altamente tecnologizados qué agencias realizan mejor los no humanos, qué agencias realizan mejor los humanos, $\mathrm{y}$ qué agencias se realizan mejor en colaboración.

\section{CONCLUSIONES}

La inminente consolidación de la cuarta revolución industrial impone el desafío de valorar en qué medida los referentes teóricos, conceptuales y metodológicos que se han usado desde hace más de cincuenta años para estudiar la tecnología educativa aún son adecuados para su análisis y desarrollo, o si ya es necesario despojarse de algunos lastres para cumplir con tal finalidad.
El aprendizaje en red es un término cuyo objetivo es definir un campo de estudio para los escenarios altamente mediados por tecnología. Sin embargo, por ser heredero de ciertos principios ontológicos y epistemológicos del humanismo moderno tiene, al menos, dos lastres conceptuales: el carácter antropocéntrico y la dicotomía reduccionista.

Las reformulaciones ontológicas del poshumanismo desvanecen esos lastres conceptuales y demuestran que lo humano y lo no humano deberían tener la misma importancia para la investigación educativa porque ambos son fundamentales al momento de aprender. Además, no es posible separar al que aprende de lo que aprende ni de las cosas con las que aprende; es decir, lo no humano es condición de posibilidad del aprendizaje y no solo un accesorio del aprendizaje. Por otra 
parte, las reformulaciones epistemológicas de la teoría del actor-red demuestran que la sociedad, en sí misma, no existe, sino que las asociaciones de elementos humanos y no humanos forman sociedades. Una de las alianzas más relevantes para la investigación educativa es el ensamblaje de elementos humanos y no humanos para aprender.

Así, una redefinición de aprendizaje en red postularía que el aprendizaje, en sí mismo, es una red de elementos y agencias heterogéneas en correcto funcionamiento. $\mathrm{El}$ aprendizaje es un proceso performativo, es decir, cuando las agencias dejan de operar, la red de aprendizaje desaparece. Por eso, el desafío y el objeto de la investigación educativa, desde este punto de vista, es reconocer que se requieren nuevos procedimientos de investigación ante la inminente consolidación de la cuarta revolución industrial. Así, es posible rastrear de manera inductiva y abductiva qué elementos humanos y no humanos se asocian para generar agencias que permitan originar redes de aprendizaje, cómo se fortalecen, cómo se asocian a otras redes y por qué desaparecen. a

\section{REFERENCIAS BIBLIOGRÁFICAS}

Adams, Samantha; Cummins, Michele; Davis, Adam; Freeman, Alex; Hall, Courtney \& Ananthanarayanan, Vanish (2017). NMC Horizon Report: 2017 Higher Education Edition. Austin, Estados Unidos: The New Media Consortium.

Arbea, Antonio. (2002). El concepto de humanitas en el Pro Archia de Cicerón. Onomázein, vol. 7, pp. 339-400. Recuperado de http://onomazein.letras.uc.cl/Articulos/7/18_Arbea.pdf

Baygin, Mehmet; Yetis, Hasan; Karakose, Mehmet \& Akin, Erhan (2016). An effect analysis of industry 4.0 to higher education. IEEE. Recuperado de http://ieeexplore.ieee.org/stamp/stamp.jsp?arnumber $=7760744$
Braidotti, Rosi. (2013). The posthuman. Cambridge, Reino Unido: Polity Press.

Bryant, Antony. (2017). Grounded theory and grounded theorizing: Pragmatism in research practice. Nueva York, Estados Unidos: Oxford University Press.

Carlson, Dennis. (2015). Foreword. En Nathan Snaza \& John A. Weaver (eds.). Posthumanism and educational research (pp. 1-6). Nueva York, Estados Unidos: Routledge.

Chernilo, Daniel. (2017). Debating humanity: Towards a philosophical sociology. Cambridge, Reino Unido: Cambridge University Press.

Daemmrich, Arthur. (2017). Invention, innovation systems, and the Fourth Industrial Revolution. Technology and Innovation, vol. 18, núm. 4, pp. 257-265. http:// dx.doi.org/10.21300/18.4.2017.257

DeLanda, Manuel. (2006). A new philosophy of society: Assemblage theory and social complexity. Londres, Reino Unido: Continuum.

Devenin, Verónica \& Henríquez, Guillermo. (2011). Narrativas tecnológicas: un ejemplo de aplicación de la sociología de las asociaciones. Cinta de Moebio: Revista de Epistemología de Ciencias Sociales, vol. 41, pp. 167-181. https://doi.org/10.4067/ S0717-554X2011000200004

Devezas, Tessaleno; Leitao, Joao \& Sarygulov, Askar. (2017). Industry 4.0: Entrepreneurship and structural change in the new digital landscape. Cham, Alemania: Springer International Publishing.

Dolphijn, Rick \& Van der Tuin, Iris. (2011). Pushing dualism to an extreme: On the philosophical impetus of a new materialism. Continental Philosophy Review, vol. 44, núm. 4, pp. 383-400. https://doi.org/10.1007/s11007-0119197-2

Dunne, Danielle \& Dougherty, Deborah. (2015). Abductive reasoning: How innovators navigate in the labyrinth of complex product innovation. Organization Studies, vol. 37, núm. 2, pp. 131-159. https://doi. org $/ 10.1177 / 0170840615604501$

Echeverría, Bolívar. (2006). El humanismo del existencialismo. Diánoia, vol. 51, núm. 57, pp. 189-199. Recuperado de http://dianoia.filosoficas.unam.mx/ files/5713/6019/3711/DIA57 Echeverria.pdf

Echeverría, Javier \& González, Marta. (2009). La teoría del actor-red y la tesis de la tecnociencia. ARBOR Ciencia, 
Pensamiento y Cultura, vol. 185, núm. 738, pp. 705-720. https://doi.org/10.3989/arbor.2009.738n1047

Escudero, Alexandro. (2016a). Aprendizaje en red: fundamentos ontoepistemológicos para su investigación. En Rosabel Roig-Vila (ed.). Tecnología, innovación e investigación en los procesos de enseñanza-aprendizaje (pp. 1609-1615). Barcelona, España: Octaedro. Recuperado de http://rua.ua.es/dspace/handle/10045/61787

Escudero, Alexandro. (2016b). El proceso inductivo de investigación en los entornos personales de aprendizaje. En Víctor Larios 0sorio, Teresa Guzmán Flores y María Teresa García Ramírez (eds.). Escenarios y desafíos de la tecnología educativa (pp. 31-50). Ciudad de México, México: Fontamara.

Espinosa, Lorenzo. (2016). Movimientos sociales. X taller internacional 10. de mayo. En A. Rodríguez y J. Castellanos (eds.). Esbozo para una categoría de humanismo en las epistemologías del sur (pp. 29-44). La Habana: Instituto de Historia de Cuba/Universidad Autónoma de Chapingo.

Fenwick, Tara \& Edwards, Richard. (2011). Introduction: Reclaiming and renewing actor network theory for educational research. Educational Philosophy and Theory, vol. 43, núm. S1, pp. 1-13. http://dx.doi. org/10.1111/j.1469-5812.2010.00667.x

Fenwick, Tara \& Edwards, Richard. (2010). Actor-network theory in education. Nueva York: Routledge.

Fenwick, Tara; Edwards, Richard \& Sawchuk, Peter. (2011). Emerging approaches to educational research. Tracing the sociomaterial. Nueva York, Estados Unidos: Routledge.

Gibson, Barry \& Hartman, Jan. (2014). Rediscovering grounded theory. Beverly Hills, Estados Unidos: Sage.

González, Sergio. (2015). Antropología y el estudio de las ontologías a principios del siglo XXI: sus problemáticas y desafíos para el análisis de la cultura. Estudios sobre las Culturas Contemporáneas, vol. 21, núm. 42, pp. 39-64. Recuperado de http://www.redalyc. org/articulo.oa?id =31642649003

Gros, Begoña. (2012). Retos y tendencias sobre el futuro de la investigación acerca del aprendizaje con tecnologías digitales. RED: Revista de Educación a Distancia, vol. 32, pp. 3-13. Recuperado de http://www. um.es/ead/red/32/gros.pdf
Harman, Graham. (2009). Prince of networks: Bruno Latour and metaphysics. Melbourne, Australia: Repress.

Hermann, Mario; Pentek, Tobias \& Otto, Boris. (2016). Design principles for industrie 4.0 scenarios: A literature review. 49th Hawaii International Conference on System Sciences Design, pp. 3928-3937. IEEE. https:// doi.org/10.1109/HICSS.2016.488

Hintz, Eric. (2011). The post-heroic generation: American independent inventors, 1900-1950. Enterprise \& Society, vol. 12, núm. 4, pp. 732-748. https://doi. org/10.1017/S146722270001065X

Holton, Judith \& Walsh, Isabelle. (2016). Classic grounded theory: Applications with qualitative and quantitative data. Thousand Oaks, Estados Unidos: Sage.

Hsu, Yu-Chen; Ho, Hsin Ning Jessie; Tsai, Chin-Chung; Hwang, Gwo-Jen; Chu, Hui-Chun; Wang, Chin-Yeh \& Chen, Nian-Shing. (2012). Research trends in technology-based learning from 2000 to 2009: A content analysis of publications in selected journals. Journal of Educational Technology \& Society, vol. 15, núm. 2, pp. 354-370. Recuperado de http://search.ebscohost. com/login. aspx?direct $=$ true\&db $=a 9 h \& A N=765592$ 51\&lang $=$ es\&site $=$ ehost-live

Hung, Jui-long. (2012). Trends of e-learning research from 2000 to 2008: Use of text mining and bibliometrics. British Journal of Educational Technology, vol. 43, núm. 1, pp. 5-16. https://doi.org/10.1111/j.14678535.2010.01144.x

Jiménez, Javier; Bustamante, Mónica \& Albornoz, María. (2015). El problema del determinismo en las políticas de educación y TIC. En H. Thomas, M. Albornoz \& F. Picabea (eds.). Políticas tecnológicas y tecnologías políticas. Dinámicas de inclusión, desarrollo e innovación en América Latina (pp. 207-240). Buenos Aires, Argentina: Flacso Ecuador/Universidad Nacional de Quilmes.

Kagermann, Henning; Wahlster, Wolfgang \& Helbig, Johannes. (2013). Securing the future of German manufacturing industry: Recommendations for implementing the strategic initiative Industrie 4.0: Final report of the Industrie 4.0 Working Group. Recuperado de http://www.acatech.de/fileadmin/ user_upload/Baumstruktur_nach_Website/Aca- 
tech/root/de/Material_fuer_Sonderseiten/Industrie_4.0/Final_report_Industrie_4.0_accessi ble.pdf

Kakkori, Leena \& Huttunen, Rauno. (2010). The Sartre-Heidegger controversy on humanism and the concept of Man in education. Educational Philosophy and Theory, vol. 43, núm. 4. https:// doi.org/10.1111/j.1469-5812.2010.00680.x

Knox, Jeremy. (2016). Posthumanism and the massive open online course: Contaminating the subject of global education. Nueva York, Estados Unidos: Routledge.

Latour, Bruno. (2009). On recalling ANT. En John Law \& John Hassard (eds.). Actor network and after (pp. 15-25). Oxford, Reino Unido: Blackwell.

Latour, Bruno. (2008). Reensamblar lo social: una introducción a la teoría del actor-red. Buenos Aires, Argentina: Manantial.

Latour, Bruno. (2007). Nunca fuimos modernos: ensayo de antropología simétrica. Buenos Aires, Argentina: Siglo XXI.

Law, John. (2004). After method: Mess in social science research. Nueva York, Estados Unidos: Routledge.

Locke, Karen; Golden-Biddle, Karen \& Feldman, Martha. (2008). Making doubt generative: Rethinking the role of doubt in the research process. Organization Science, vol. 19, núm. 6, pp. 907-918. https://doi.org/10.1287/orsc.1080.0398

Lorenz, Markus; Kuepper, Daniel; Ruessmann, Michael; Heidemann, Ailke \& Bause, Alexandra. (2016). Time to accelerate in the race toward industry 4.0. Boston, Estados Unidos: The Boston Consulting Group. Recuperado de https://www.bcgperspectives. com/Images/BCG-Time-to-Accelerate-in-the-Race-TowardIndustry-4.0-May-2016_tcm80-211060.pdf

Lorenz, Markus; Ruessmann, Michael; Strack, Rainer; Lueth, Knud \& Bolle, Moritz. (2015). Man and machine in industry 4.0: How will technology transform the industrial workforce through 2025. Boston, Estados Unidos: The Boston Consulting Group.

MacLeod, Roy. (2016). Scientists. En J. Winter (ed.). Cambridge history of the First World War (pp. 434-459). Reino Unido: Cambridge University Press.

Minerva, Roberto; Biru, Abyi \& Rotondi, Domenico. (2015). Towards a definition of the Internet of things (IoT). Turín, Italia: IEEE.

Morita-Alexander, Adelina; García-Ramírez, María Teresa \& EscuderoNahón, Alexandro. (2016). Análisis de la percepción de las competencias genéricas en instituciones de educación superior en México. Revista de Educación y Desarrollo, vol. 38, pp. 69-78. Recuperado de http://www.cucs.udg.mx/revistas/edu_desarroIlo/anteriores/38/38_Morita.pdf
OCDE. (2017). OECD Skills strategy diagnostic report. Ciudad de México, México/París, Francia: OECDE.

Pape, Helmut. (1999). Abduction and the topology of human cognition. Transactions of the Charles S. Peirce Society, vol. 453, núm. 2, pp. 248-269. Recuperado de http://www.jstor.org/stable/10.2307/40320760

Pedersen, Helena. (2010). Is "the posthuman" educable? On the convergence of educational philosophy, animal studies, and posthumanist theory. Discourse: Studies in the Cultural Politics of Education, vol. 31, núm. 2, pp. 237-250. http://dx.doi. org/10.1080/01596301003679750

Pignuoli-Ocampo, Sergio. (2014). La posición epistemológica del constructivismo simétrico de Bruno Latour. Cinta de Moebio. Revista de Epistemología de Ciencias Sociales, vol. 52, núm. marzo, pp. 91-103. http://dx.doi.org/10.4067/S0717$554 \times 2015000100008$

Reichertz, J0. (2007). Abduction: The logic of discovery of Grounded Theory. En A. Bryant \& K. Charmaz (eds.). The SAGE handbook of Grounded Theory (pp. 214-228). Londres, Reino Unido: Sage Rivas, Axel \& Delgado, Lucas Esteban. (2016). Un mapa del futuro: 50 innovaciones educativas en América Latina. Washington, DC, Estados Unidos: Graduate XXI. Recuperado de https://publications.iadb.org/handle/11319/7595

Roland, Berger. (2016). España 4.0: el reto de la transformación digital de la economía. Madrid, España: Siemens. Recuperado de https://w5.siemens.com/spain/web/es/estudiodigitalizacion/ Documents/Estudio_Digitalizacion_Espana40_Siemens.pdf Rose, Gideon. (2016). The Fourth Industrial Revolution: A Davos reader. Nueva York, Estados Unidos: Council on Foreign Relations.

Schwab, Klaus. (2016). The Fourth Industrial Revolution. Colonia, Alemania/Ginebra, Suiza: World Economic Forum.

Schwab, Klaus. (2015). The fourth industrial revolution: What it means, how to respond. Recuperado de https://www.foreignaffairs.com/articles/2015-12-12/fourth-industrial-revolution

Slama, Dirk; Puhlmann, Frank; Morrish, Jim \& Bhatnagar, Rishi. (2015). Enterprise IoT: Strategies and Best practices for connected products and services. Sebastopol, Estados Unidos: 0'Reilly Media.

Snaza, Nathan \& Weaver, John. (eds.). (2015). Posthumanism and educational research. Nueva York, Estados Unidos: Routledge.

St. Pierre, Elizabeth. (2013). The posts continue: becoming. International Journal of Qualitative Studies in Education, vol. 26, núm. 6, pp. 646-657. https://doi.org/10.1080/09518398.2013 .788754 
Tecnalia. (2017). Recuperado de http://www.tecnalia.com/es/

Thomas, Hernán \& Buch, Alfonso. (2013). Actos, actores y artefactos. Sociología de la tecnología. Bernal, Argentina: Universidad Nacional de Quilmes. Recuperado de http://www.unq.edu.ar/ advf/documentos/520933c153e48.pdf

UNESCO. (2015). UNESCO Science report-towards 2030. París, Francia: UNESCO.

Usher, Robin \& Edwards, Richard. (2005). Subjects, networks and positions: Thinking educational guidance differently. British Journal of Guidance \& Counselling, vol. 33, núm. 3, pp. 397-410. https:// doi.org/10.1080/03069880500179640
Weaver, John. (2010). The posthuman condition: A complicated conversation. En Erik Malewski (ed.). Curriculum Studies Handbook: The next moment (pp. 190.200). Nueva York, Estados Unidos: Routledge.

Wolfe, Cary. (2010). What is posthumanism? Estados Unidos: University of Minnesota Press.

World Economic Forum. (2016). The future of jobs. Employment, Skills and workforce strategy for the Fourth Industrial Revolution. Recuperado de http://www3.weforum.org/docs/WEF_Future_of_Jobs.pdf

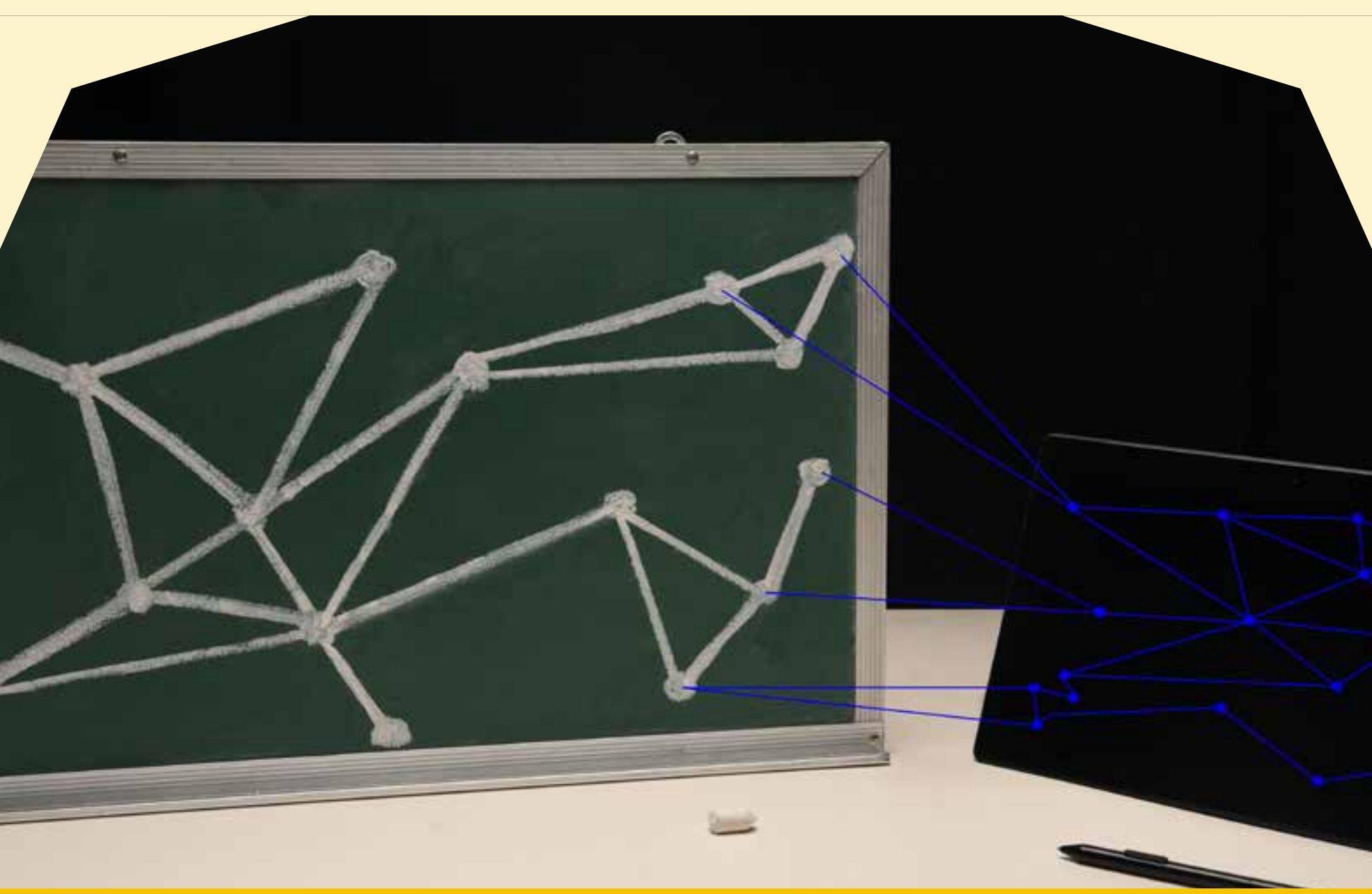

"Este artículo es de acceso abierto. Los usuarios pueden leer, descargar, distribuir, imprimir y enlazar al texto completo, siempre y cuando sea sin fines de lucro y se cite la fuente."

\section{CÓMO CITAR ESTE ARTíCULO:}

Escudero Nahón, Alexandro. (2018). Redefinición del “aprendizaje en red” en la cuarta revolución industrial. Apertura, 10 (1), pp. 149-163. http://dx.doi.org/10.32870/Ap.v10n1.1140 